\title{
Die Alpha-Funktion eines analytischen Behandlungsteams
}

\author{
Colette Pfistner (Basel)
}

Zusammenfassung: Die Psychotherapeutische Tagesklinik (PTK) ist ein Angebot des Zentrums für Psychosomatik und Psychotherapie (ZPP) der Universitären Psychiatrischen Kliniken Basel (UPK) und verfügt über 16 Behandlungsplätze. Das interdisziplinäre Behandlungsteam arbeitet mit einem psychodynamischen Behandlungskonzept. Durch ein relativ starres Behandlungsgerüst sowie verschiedene Gruppenangebote und Milieutherapie, aber auch in den Einzeltherapien soll eine Reaktivierung von unbewussten Konflikten und interaktionellen Schwierigkeiten der Patient*innen ermöglicht werden. Diese können nun in einem geschützten therapeutischen Rahmen neu bearbeitet werden, was zu korrektiven Erfahrungen führt und Veränderungen ermöglicht. Die vorliegende Arbeit setzt sich dabei speziell mit den Funktionen des Behandlungsteams sowie der Arbeit in Abwesenheit der Patient*innen auseinander. Anhand eines Fallbeispiels wird gezeigt, wie eine psychoanalytische Haltung innerhalb eines interdisziplinären Therapeutenteams dabei hilft, im verbalen Austausch miteinander ein gemeinsames Verstehen zu erarbeiten und wie dadurch Verbindungen zwischen unintegrierten Aspekten des Seelischen der Patient*innen hergestellt werden können. Dabei wird mit Bezug auf Bion der Prozess, der innerhalb des Teams geschieht, als «Alpha-Funktion des Teams» bezeichnet.

Schlüsselwörter: Teilstationäre Psychotherapie, Reflexionsraum, analytisches Behandlungsteam, Alpha-Funktion des Teams.

Im Rahmen meiner Tätigkeit als Oberärztin einer psychoanalytisch ausgerichteten Tagesklinik sehe ich jährlich zahlreiche Patientinnen und Patienten (Patient*innen) unter anderem zum Ein- und zum Austrittsgespräch. Dabei fallen mir sehr unterschiedliche Entwicklungen auf, welche sicherlich überwiegend von patientenspezifischen Faktoren abhängen. Als Abteilungsleitung beschäftigt mich jedoch auch die Frage, welche Faktoren unserer Behandlungen wirksam oder allenfalls schädlich sind. Insbesondere interessiert mich die Funktion des 
Austausches innerhalb des Behandlungsteams in Form von Rapporten, Sitzungen und Patientenbesprechungen. Die Arbeit in Abwesenheit der Patient*innen ist sehr zeitintensiv und man könnte argumentieren, dass dabei kostbare Zeitressourcen verschwendet werden. Die vorliegende Arbeit setzt sich daher mit der Frage auseinander, welche Wirkung oder Funktion das interdisziplinäre Behandlungsteam in Abwesenheit der Patient*innen haben könnte.

Die Psychotherapeutische Tagesklinik (PTK) der Universitären Psychiatrischen Kliniken (UPK) Basel nahm im Jahr 1991 ihre Arbeit mit initial 10 Behandlungsplätzen auf und konnte später auf die ursprünglich vorgesehenen 16 Behandlungsplätze erweitert werden. Im 1998 erschienenen Buch «Teilstationäre Psychotherapie - Theorie und Praxis» erzählt Joachim Küchenhoff die Entstehungsgeschichte der Basler PTK (Küchenhoff, 1998). Der erste Teil «Grundlagen und Modelle der teilstationären Psychotherapie» widmet sich mitunter der «Theorie und Praxis der teilstationären Behandlung» sowie dem «Team als Behandlungssubjekt». Das damals etablierte Therapieprogramm mit den für alle Patient*innen verbindlichen Präsenzzeiten von Montag bis Freitag jeweils von 8.30 bis $17.00 \mathrm{Uhr}$ - inklusive der «ausführlichen Patientendiskussion» im Behandlungsteam am Donnerstagnachmittag - wird noch heute in leicht modernisierter Form zumeist erfolgreich umgesetzt. Im zweiten Teil des oben genannten Buches werden unter dem Titel «Praxis der teilstationären Psychotherapie» die wichtigsten der noch heute praktizierten Therapieangebote beschrieben. Im dritten Teil stellen C. Kern und J. Küchenhoff die ersten empirischen Daten zur teilstationären Psychotherapie vor.

Noch heute verfügt die PTK über 16 Behandlungsplätze. Die Behandlungsdauer beträgt entweder 6 Wochen oder 18 Wochen. Die Ein- und Austrittstermine werden im Voraus festgelegt und sind somit von Anfang an bekannt. Der zeitliche Rahmen wird damit klar vorgegeben. Nach einer Zuweisung erfolgen zuerst zwei bis drei Vorgespräche in der Ambulanz des Zentrums für Psychosomatik und Psychotherapie (ZPP). Dort wird die Indikation für eine PTK-Behandlung nach Rücksprache mit der Oberärztin geklärt. Dabei werden die Therapiebedürftigkeit, die Therapiemotivation sowie die Therapiefähigkeit der angemeldeten Patient*innen so gut als möglich abgeschätzt (Bürgin \& Steck, 2013). Dadurch sollen Therapieabbrüche, Wirkungslosigkeit oder gar negative Effekte als Folge der Behandlung minimiert werden. Das Spektrum der in der PTK behandelten psychischen Erkrankungen ist relativ breit. Die meisten Patient*innen leiden jedoch an einer depressiven Symptomatik in Kombination mit leichten bis mittelgradigen Schwächen in der Persönlichkeitsstruktur. An äusseren Faktoren wird lediglich 
eine geklärteWohnsituation im Kanton Baselstadt vorausgesetzt. Die Vorgespräche dienen mitunter der Abschätzung, ob die zugewiesenen Patient*innen im aktuellen Zustand dazu fähig sind, den tagesklinischen Rahmen inklusive der täglichen Präsenzzeiten einzuhalten. Als kontraindiziert erachten wir akute Psychosen, akute, handlungsnahe Suizidalität sowie aktive Suchterkrankungen.

Das heutige PTK-Team besteht im Kern aus einer Oberärztin, einem in der Facharztausbildung fortgeschrittenen Assistenzarzt, einer Fachpsychologin, einer Assistenzpsychologin, einer Kunsttherapeutin, einer Musiktherapeutin, einer Sozialarbeiterin (30\%) sowie vier erfahrenen Pflegefachfrauen. Die pflegerische Abteilungsleitung bildet zusammen mit mir, der abteilungsleitenden Oberärztin, eine sogenannte «Tandem-Leitung». Der/die Einzeltherapeut*in sowie die Pflegerische Bezugsperson begleiten den/die Patient*in über die ganze Behandlungsdauer hinweg und bilden zusammen das sogenannte Kernteam. Die Mitarbeitenden des engeren Behandlungsteams sind allesamt psychoanalytisch geschult und mit psychodynamischen Konzeptualisierungen, insbesondere auch mit der Operationalisierten Psychodynamischen Diagnostik (OPD-2), vertraut (Arbeitskreis OPD \& Cierpka, 2014).

Die Besprechungen des Behandlungsteams in Abwesenheit der Patient*innen nehmen insgesamt viel Zeit in Anspruch: Sie finden jeweils vor und nach den diversen Therapieangeboten in festgelegten Konstellationen statt. Zusätzlich gibt es regelmässige Besprechungen zwischen einzelnen Teammitgliedern sowie Treffen, welche vorwiegend dem Austausch von Anliegen des Behandlungsteams dienen. Eine Bündelung der Informationen erfolgt in den ausführlichen Patientenbesprechungen mit Fokusbildung. Für Patient*innen im kurzen 6-WochenSetting erfolgt diese nach den ersten zwei Wochen, für Patient*innen im langen 18-Wochen-Setting nach drei sowie nach neun Wochen. Der dabei festgelegte Fokus wird mit den Patient*innen rückbesprochen und gilt für das gesamte Behandlungsteam als Orientierungshilfe, respektive als gemeinsames Ziel, das die verschiedenen Therapieangebote über unterschiedliche Wege erreichen.

Durch die analytische Ausrichtung aller Teammitglieder, den klar vorgegebenen Rahmen sowie die verschiedenen Gefässe, welche dem interdisziplinären Austausch im Team dienen, entsteht eine Art Reflexionsraum. Darin werden die mit unseren Patient*innen gemachten Erlebnisse, Wahrnehmungen und Beobachtungen in Worte gefasst und zusammengetragen, werden Übertragungsund Gegenübertragungsgefühle ausgetauscht. Überdies werden Dynamiken, welche sich dem Team zeigen, sowie die variierenden psychoanalytischen Haltungen, 
die das Team einnehmen möchte, diskutiert. Es ist ein Raum, in dem das, was schon da ist in der Nachträglichkeit gemeinsam reflektiert und verbalisiert werden kann.

Hierbei kann die Patientengruppe im Zentrum der Beobachtung stehen oder aber auch das Behandlungsteam der PTK. Dabei lassen sich z. B. parallele Dynamiken bei Patienten und Team entdecken, aber auch situativ unterschiedliche Interaktionen, Vorgehensweisen, Diskussionen oder Abweichungen von Standards innerhalb des Behandlungsteams. So kommt das Team im Falle von Verletzungen des therapeutischen Rahmens bei einigen Patient*innen relativ schnell und nach kurzer Diskussion zu einer klaren Haltung, wie mit der Situation weiter umgegangen werden soll, während andere Patient*innen wiederholt kontroverse Meinungen im Team über die Haltung und das weitere Vorgehen auslösen. Solche unterschiedlichen Dynamiken versuchen alle Teammitglieder wahrzunehmen und zu benennen, um sie als eine mögliche Ausdrucksform des «psychodynamisch Unbewussten, konkretisiert als unbewusste Konflikte, Abwehrmechanismen, Symptombildungen, Phantasien, Bewusstseinszuständen, Modi derVerarbeitung von Konflikten und Traumata oder Transformationen» zu verstehen (Mertens, 2014, S. 756).

Eine Aufgabe der Oberärztin ist es, dafür zu sorgen, dass das Team sich so oft als möglich im Arbeitsmodus befindet und als «Arbeitsgruppe» funktioniert (Bion, 1961). Bion bezeichnet die Arbeitsgruppentätigkeit, also die «geistige Betätigung, die darauf abzielt, der vorliegenden Aufgabe zu dienen», als eine Eigenschaft jeder Gruppe von Individuen, die zu Arbeitszwecken zusammengekommen sind. Diese Bestrebungen würden jedoch auch durch affektive Triebkräfte beeinflusst, welche aus drei Grundannahmen über ihre unbewussten Ziele (Bewältigung von Abhängigkeit, Paarbildung, Kampf oder Flucht) entspringen.

Natürlich helfen auch die monatliche Teamsupervision sowie die externen Supervisionen der Einzeltherapeut*innen dabei, als Team funktionsfähig zu bleiben. Vorstellungen von dem, was geschieht und was das Team zu erkennen glaubt, bedürfen manchmal einer Korrektur von aussen, um aus den verschiedenen rollenbedingten Grundannahmen der Einzelmitglieder immer wieder gemeinsam in den Funktionsmodus einer Arbeitsgruppe zurückzukehren. Das Team scheint einem Organismus zu gleichen, der sich durch das Benennen des Nicht-Funktionierens immer wieder selbst repariert. Und durch die gemeinsame Reflexion dessen, was die Patient*innen dem Team im Laufe der Therapie präsentieren, was sie im Team auslösen und wie das Team damit umgeht, geschieht eine Art Verdauungsprozess. So kann zum Beispiel eine Diskussion, welche durch eine Identifikation der einzelnen Teammitglieder mit unterschiedlichen, voneinan- 
der abgespaltenen Anteilen eines Patienten ausgelöst wurde, dabei helfen, diese Teile zu erkennen. Mitunter kann das Benennen dieser Spaltung dem Team dabei helfen, sich wieder zu versöhnen und in den Arbeitsmodus zurückzukehren. Im Arbeitsmodus kann das Team seine psychische Verdauungsfunktion wahrnehmen und es dem Patienten durch die Integrationsleistung derVersöhnung ermöglichen, selbst erste Verbindungen zwischen unintegrierten Aspekten des Seelischen herzustellen. Diesen Prozess, der innerhalb des Teams geschieht, möchte ich mit Bezug auf Bion die «Alpha-Funktion des Teams» nennen: Beta-Elemente, mit denen man nicht denken kann, werden umgewandelt zu Alpha-Elementen, mit denen man denken und fühlen kann (Vermote, 2019). Die folgende Fallvignette soll dabei helfen, diese Hypothese etwas zu veranschaulichen:

Der 23-jährige Herr S. wurde uns zugewiesen mit einer depressiven Symptomatik und sozialen Ängsten mit ausgeprägtem sozialem Rückzug. Eine durch die Schweizerische Invalidenversicherung (IV) finanzierte Ausbildung wurde kurz zuvor abgebrochen. Herr S. hatte zu viele Fehlzeiten, war zu langsam und konnte die geforderte Leistung nicht erbringen. Bereits im Rahmen der Vorgespräche für die tagesklinische Behandlung löste der sehr jungenhafte, scheue und wortkarge Herr S. durch seine Unbeholfenheit sowie seine berührende Biografie den Impuls aus, ihm Unterstützung anzubieten. Aus ambulanten Vorberichten war bekannt, dass der Vater des Patienten die Familie überraschend verlassen und den Kontakt vollständig abgebrochen hatte, als Herr S. zwölf Jahre alt war. Als Folge davon habe die tablettensüchtige Mutter eine schwere Depression entwickelt, dabei jedoch sämtliche Hilfsangebote abgelehnt. So habe Herr S. als Teenager den Haushalt inklusive der Versorgung der hauptsächlich im Bett liegenden Mutter alleine bewerkstelligen müssen. Gegen Ende seiner regulären Schulzeit habe der Patient ebenfalls das Haus kaum noch verlassen und zunehmend soziale Ängste entwickelt. Aufgrund von schulischen Problemen kam es zu ersten psychiatrischen Kontakten, in denen sich Herr S. jedoch ohne Leidensdruck präsentierte. Erst im Rahmen einer längeren stationären Behandlung der Mutter erhielt der damals 20-jährige Patient wegen finanzieller Schwierigkeiten eine erste Unterstützung durch die Sozialhilfe sowie einen Platz in einer Wohngruppe. Diese hatte er zwei Jahre später jedoch wieder verlassen, weil er lieber alleine wohnen wollte.

Auch im Rahmen der Indikationsbesprechung löste der Bericht über die Vorgeschichte von Herrn S. bei den Anwesenden den Wunsch aus, ihm etwas anzubieten und ihm zu helfen. Gewisse Vorbehalte lösten seine fragliche Therapiemotivation sowie die vorhersehbaren Verletzungen des therapeutischen Rahmens, eines unserer wichtigsten Werkzeuge, aus. Aufgrund seiner Symptomatik mussten 
wir bei einer teilstationären Behandlung von Herrn S. mit Störungen des Rahmens in Form von Fehlzeiten rechnen, welche dann jeweils prioritär behandelt und besprochen werden müssten. In der Regel empfehlen wir Patient*innen, bei denen es von Anfang an klar ist, dass sie den intensiven therapeutischen Rahmen der PTK nicht zuverlässig einhalten können oder dadurch überfordert werden, eher eine stationäre Therapie oder eine sozialpsychiatrisch ausgerichtete Tagesstruktur mit einem weniger intensiven Therapieprogramm sowie einer weniger strengen Anwesenheitspflicht. Bereits im Vorfeld lehnte Herr S. ein stationäres Angebot jedoch klar ab. Aufgrund seiner sozialen Ängste brauche er seine eigene Wohnung als Rückzugs- und Regenerationsort. Die Teilnehmenden der Indikationssitzung waren sich einig, dass Herr S. sich ohne Therapie weiter zuhause zurückziehen und günstigstenfalls irgendwann eine IV-Rente erhalten oder auch verwahrlosen würde. Durch die fehlenden Alternativen wurde der Druck spürbar, den Herr S. auslöste. Im Sinne einer Chance zur Nachreifung wurde er in das 18-wöchige Programm der PTK aufgenommen. Ein eher aussergewöhnliches Therapieziel bestand in einer regelmässigen Teilnahme am Therapieprogramm. Ein weiteres Behandlungsziel war die Verbesserung der Kommunikation, sowohl nach aussen als auch nach innen. Es fiel Herrn S. äusserst schwer, sich zu öffnen und über seine Gefühle zu reden. Bereits zu Beginn der Behandlung wurde eine starke Ambivalenz des Patienten hinsichtlich der Therapie deutlich. In der Beziehungsdynamik erlebte Herr S. andere als bedrohlich übergriffig, was dazu führte, dass er sich zurückzog und seinen Ärger gegen sich selbst richtete oder sich passiv-aggressiv verhielt, z. B. verschlief oder nicht an der Therapie teilnahm. Dies führte zu vermehrten Versorgungsangeboten, welche er wiederum als intrusiv erlebte. Über eine lange Zeit der Behandlung lehnte Herr S. mögliche Hilfestellungen, wie z. B. eine psychopharmakologische Unterstützung, ab. Im Behandlungsteam der PTK löste er wiederholt intensive Diskussionen sowie eine Spaltung aus. Der eine Teil der Teammitglieder wollte dem hilfebedürftigen jungen Mann die bestmöglichen Bedingungen zur Nachreifung anbieten. Der andere Teil des Teams wies auf die zahlreichen Rahmenverletzungen hin und forderte Konsequenzen in Form eines Behandlungsabbruchs. Von der Patientengruppe wurde der ruhige und zurückgezogene Herr S. aufgrund seiner Beobachtungsgabe sowie seiner feinfühligen, treffenden Kommentare und Feedbacks insbesondere in der Musik- sowie in der Kunsttherapie zunehmend geschätzt. Vor allem in der Musiktherapie gelang es dem Patienten, sich zu öffnen, Neues auszuprobieren und die dort entstehenden Interaktionen innerhalb der Gruppe sowie seine oft taktangebende Rolle im Hintergrund zu geniessen. Musik hatte eine besondere Bedeutung für den Patienten. 
Sie schien seine Ängste etwas zu beruhigen und ihm eine Möglichkeit zum Rückzug, wie auch zur Interaktion zu bieten. Entsprechend gross war auch der Einsatz der Musiktherapeutin. In den Diskussionen innerhalb des Behandlungsteams setzte sie sich wiederholt für eine Weiterbehandlung des Patienten ein. Zu einer ersten Zuspitzung der Meinungsverschiedenheiten im Team kam es nach etwa 12 Wochen der ersten PTK-Behandlung. Die bereits zahlreichen Absenzen sowie Verspätungen des Patienten nahmen eher zu als ab. Zudem schien seine Teilnahme an den Therapien selektiv zu werden mit einer Vermeidung der morgendlichen Gesprächsgruppen durch Verspätung, aber auch Rückzug in andere Räume der PTK. Der eine Teil des Teams erachtete die gehäuften Rahmenverletzungen als einen Ausdruck einer negativen therapeutischen Reaktion, welche nun mit dem Patienten bearbeitet werden sollte. Der andere Teil des Teams sah darin eine weitere Abnahme des Engagements und der Therapiemotivation des Patienten und plädierte für einen Abbruch der Behandlung. Das Team einigte sich darauf, Herrn S. nun über einen drohenden Therapieabbruch zu informieren, sollte sich seine Pünktlichkeit nicht wieder verbessern. Zur Unterstützung wurden ihm sehr konkrete Hilfsangebote gemacht, wie etwa ein Weckdienst, eine Begleitung auf dem Weg zu uns in die PTK oder die Übernahme von Telefonaten mit der pflegerischen Bezugsperson. Überdies wurde ihm erneut auch eine Psychopharmakotherapie angeboten. Nichts davon nahm er in Anspruch, lehnte aber auch nie klar ab. In den konfrontierenden Gesprächen wirkte er sehr beschämt und äusserst schweigsam. Er beantwortete knapp die ihm gestellten Fragen mit jeweils auffallend langen Antwortlatenzen und schien deutlich belastet. Die Spaltung im Team hatte inzwischen an Heftigkeit zugenommen und schien nur noch durch einen Therapieabbruch wieder auflösbar. Als Herr S. über den frühzeitigen Abbruch der Behandlung von Seitens der PTK informiert werden sollte, gelang es dem Patienten erstmals klar und mit einer gewissen Vehemenz seinen Wunsch zu benennen, die Behandlung in der PTK fortzusetzen. Er sei nun auch dazu bereit, das ihm mehrfach angebotene Antidepressivum einzunehmen und Hilfsangebote in Anspruch zu nehmen. Beeindruckt vom erstmals so deutlich geäusserten Wunsch des Patienten sowie seiner Bereitschaft, Hilfe anzunehmen, traf ich die oberärztliche Entscheidung, die Behandlung weiterzuführen. Dieses Entgegenkommen wurde von einigen Teammitgliedern als ein Einknicken oder gar als Verrat der gemeinsamen, klaren Haltung gesehen. Es erforderte einige Nachbesprechungen im Team, um die aufkommenden Wogen zu glätten und ein gemeinsames Verstehen zu ermöglichen. Beide Seiten mussten dafür ein Stück weit von ihren gefestigten Positionen loskommen, auf die anderen Teammitglieder zugehen und im Gespräch 
miteinander eine neue gemeinsame Position einnehmen. Dem Patienten gelang es danach einerseits, in der Einzeltherapie seine Beziehungswünsche sowie seinen Wunsch nach einem Feedback der Patientengruppe zur Sprache zu bringen, als auch andererseits zuverlässiger an der Therapie teilzunehmen. Als der reguläre Austrittstermin näher rückte und der bevorstehende Abschied konkreter wurde Herr S. hatte gemeinsam mit der pflegerischen Bezugsperson ein Formular mit den notwendigen Schritten in der Austrittsplanung ausgefüllt-meldete der Patient sich aufgrund von Bauchschmerzen und Übelkeit ab. Seine Mühe mit dem Aufgleisen von Anschlusslösungen konnte in den Einzelgesprächen, aber auch in der Gesprächsgruppe zum Thema werden. Insgesamt zeigte Herr S. über die letzten Wochen eine deutlich höhere Präsenz und mehr Kontakt zu Mitpatient*innen innerhalb und ausserhalb der Therapien. Bei Austritt, also nach 18 Wochen der Behandlung, schien er zwar stabilisiert, jedoch noch nicht dazu bereit, die Anforderungen für eine Ausbildung zu erfüllen. So erstaunte es uns auch nicht, als Herr S. sich einige Monate später erneut bei uns meldete mit dem Wunsch nach einer weiteren Behandlung in der PTK. Er wirkte zwar äusserlich etwas verwahrlost, war im Gespräch nun aber deutlich aktiver, sprach mehrere Sätze hintereinander im Fluss und konnte seine Gedankengänge in Worte fassen. Es sei seine Idee gewesen, sich wieder bei uns zu melden. Er erzählte, dass er sich nach Abschluss der Behandlung bei uns weder bei seiner ambulanten Therapeutin gemeldet noch die aufgegleiste Tagesstruktur wahrgenommen habe. Das Antidepressivum habe er abgesetzt, weil sein Rezept abgelaufen sei und er habe sich wieder vermehrt zuhause zurückgezogen. Wir fanden heraus, dass der Versuch einer Ausbildung über die IV inzwischen als gescheitert aufgegeben und sein «Fall» geschlossen worden war. Im PTK-Team bestand nach wie vor der Wunsch, Herrn S. etwas zu ermöglichen und ihm eine zweite Chance zu geben - unter Vorbehalt einzelner Teammitglieder, welche für ein härteres Durchsetzen der Regeln plädierten, worauf wir uns schliesslich einigten. Herr S. zögerte den Eintritt noch etwas hinaus, um einen überfälligen Diensteinsatz im Militär inklusive zweier Übernachtungen in einem Massenlager zu leisten, was ihm dann offenbar problemlos gelang. $\mathrm{Zu}$ Beginn der Behandlung bei uns schien er Mühe zu haben, mit den neuen Mitpatient*innen in Kontakt zu kommen. Seine Angst vor Zurückweisung oder Ablehnung konnte er in den Einzelsitzungen nun aber in Worte fassen. Erneut entwickelte Herr S. körperliche Beschwerden. Er nahm dennoch an den Therapien teil und löste dabei aber sowohl im Team als auch bei den Mitpatient*innen Besorgnis aus. Sein reduzierter Allgemeinzustand sowie sein Gewichtsverlust wurden derart alarmierend, dass wir uns dazu gezwungen sahen, aktiv zu werden. Wir 
thematisierten unseren Eindruck einer Überforderung im teilstationären Rahmen und legten ihm eine stationäre Behandlung nahe. Erneut lehnte Herr S. eine Verlegung ab, äusserte nun aber erstmals seinen Ärger über die Rigidität der PTK. Die Diskussionen im Team spitzten sich wieder zu. Der eine Teil äusserte den Eindruck, dass Herr S. von der PTK-Behandlung profitierte und betonte, dass es ihm stets besser gelinge, seine Wünsche wahrzunehmen und zu formulieren. Der andere Teil des Teams versuchte den Rahmen zu schützen, auch im Interesse der Mitpatient*innen, für welche der Rahmen ein wichtiger Orientierungspunkt und eine haltgebende Struktur darstellte. Dieser Teil des Teams betonte eher die Notwendigkeit, die Funktion des fehlenden Vaters wahrzunehmen und den Patienten durch klare Grenzen zu schützen. Bei mir löste er die Phantasie aus, die aktuelle Verschlechterung könnte im Sinne eines «szenischen Verstehens» ein Ausdruck seiner Traumatisierung sein, in der er nun unbewusst die Rolle seiner Mutter einnahm und uns seine frühere Ohnmacht fühlen liess (Argelander, 1970; Mertens, 2014). Nun könnte er uns dabei zusehen, wie wir mit der Situation umgehen. Eine gewisse Mitbestimmung über seinen Rahmen könnte für ihn notwendig sein, um sich der Situation respektive uns nicht ohnmächtig ausgeliefert zu fühlen.

Herr S. selbst setzte sich erneut unter Aushandlung eines konkreten Massnahmen-Plans für die Weiterbehandlung bei uns ein, wobei er wiederum an der Umsetzung seiner Pläne scheiterte. Das Team hatte es dank einiger Nachbesprechungen und der Reflexion der Ereignisse im Zusammenhang mit Herrn S. inzwischen wieder geschafft, sich zu versöhnen und sich darauf zu einigen, die Behandlung in der PTK noch bis zur Aufgleisung einer konkreten Alternative weiterzuführen. Parallel dazu verschwanden die körperlichen Beschwerden des Patienten. Er konnte wieder essen und sein Gewicht normalisierte sich zusehends. In den darauffolgenden Wochen drehte sich die Therapie hauptsächlich um die Aufgleisung von Anschlusslösungen, welche Herr S. nach Wahrnehmung von Besichtigungsterminen jeweils sanft, aber bestimmt ablehnte. Der Patient begann sich für eine reguläre Beendigung der 18Wochen sowie für einen Verbleib in seiner Wohnung einzusetzen. Es fanden zahlreiche Termine mit der Wohnbegleitung, dem Beistand und der IV statt und es wurde hauptsächlich an konkreten Themen gearbeitet. Herrn S. gelang es nun auch, deutlich zuverlässiger am Programm teilzunehmen. Gegen Ende der Behandlung verliebte er sich in eine Mitpatientin. Herr S. begann sich äusserlich mehr zu pflegen und sich modischer zu kleiden. Die Therapiesitzungen drehten sich vermehrt um seine unerwiderte Liebe und die damit verbundenen Gefühle. Schmerzhaftes konnte nun auch im Zusammenhang mit dem Abschied zur Sprache kommen. Am Ende war es ihm zum zweiten Mal 
gelungen, das 18-wöchige Programm der PTK regulär abzuschliessen. Diesmal waren die Anschlusslösungen aufgegleist und zuvor schon getestet. Herr S. kam seinem Ziel, eine Berufsausbildung über die IV zu absolvieren, wieder etwas näher.

Zusammenfassend lässt sich die Alpha-Funktion des analytischen Behandlungsteams wie folgt erklären: Es besteht ein relativ starres Behandlungsgerüst innerhalb dessen sich die Dynamiken der Patient*innen zeigen können. Unweigerlich inszeniert auch das interdisziplinäre Team dabei im Sinne von projektiven Identifikationen die Konflikte der Patient*innen. Ein psychoanalytisch geschultes Team wird jedoch gemeinsam im Austausch miteinander diese Dynamiken zu verstehen versuchen. Dabei wirkt dieser Prozess mit dem Ziel zu verstehen wie ein Verdauungsprozess von Beta-Elementen, welche, weil im Team alphabetisiert, den Patient*innen in verdauter Form zurückgegeben werden können (Ferro, 2007). Dies wiederum ermöglicht den Patient*innen neue Schritte im Therapieprozess.

\section{Literatur}

Arbeitskreis OPD (Hg.), Cierpka, M. (Hg.). (2014). Operationalisierte Psychodynamische Diagnostik: Das Manual für Diagnostik und Therapieplanung. Bern: Hans Huber.

Argelander, H. (1970). Das Erstinterview in der Psychotherapie. Darmstadt: WBG. Bion, W. R. (1961). Experiences in Groups and Other Papers. London: Tavistock. Bürgin, D., Steck, B. (2013). Indikation psychoanalytischer Psychotherapie bei und Jugendlichen. Stuttgart: Klett-Cotta.

Ferro, A. (2007). Klinische Implikationen von Bions Denken. Internationale Psychoanalyse 2, 151-171.

Küchenhoff, J. (1998). Teilstationäre Psychotherapie. Stuttgart; New York: Schattauer. Mertens, W. (Hg.). (2014). Handbuch psychoanalytischer Grundbegriffe. Stuttgart: Kohlhammer.

Vermote, R. (2019). Reading Bion. London; New York: Routledge.

\section{Angaben zur Autorin}

Colette Pfistner, Dr. med., 1972, geboren und aufgewachsen in Basel (Schweiz). Studium der Wirtschaftswissenschaften sowie der Humanmedizin in Basel (Arztdiplom 2001). Von 2004 bis 2010 Aufenthalt in den Niederlanden mit diversen beruflichen Tätigkeiten. Promotion an der Medizinischen Fakultät Basel 2009. Facharztausbildung in Psychiatrie und psychoanalytischer Psychotherapie in Liestal und Basel von 2011 bis 2018. Seit 2017 Oberärztin und Abteilungsleitung der PTK, Universitäre Psychiatrische Kliniken Basel. 